\title{
Clinical value of diagnosing aortico-left ventricular tunnel by echocardiography
}

\author{
MANYING XIE ${ }^{1}$, JIABAO YIN $^{1}$, QING LV $^{2}$ and JING WANG ${ }^{2}$ \\ ${ }^{1}$ Department of Ultrasound Imaging, Xiangyang Hospital Affiliated to Hubei University of Medicine, \\ Xiangyang, Hubei 441000; ${ }^{2}$ Department of Ultrasound Imaging, Xiehe Hospital Affiliated to \\ Tongji Medical College, Huazhong University of Science and Technology, Wuhan, Hubei 430022, P.R. China
}

Received February 8, 2016; Accepted September 20, 2016

DOI: $10.3892 /$ etm.2016.3747

\begin{abstract}
In order to assess the clinical value of echocardiography in the diagnosis of aortico-left ventricular tunnel (ALVT) the echocardiographic data and clinical manifestations of six patients with ALVT were analyzed in the present study. Based on the results, a comprehensive study was conducted regarding 147 cases from related documents. Six patients were confirmed with ALVT by surgery. The echocardiographic images of the patients revealed an abnormal communication beginning in the ascending aorta above the coronary arteries, bypassing the aortic valve, and ending in the left ventricle. The color Doppler flow imaging showed a high-speed to- and -fro flow with or without the involvement of the aortic valve. According to Hovaguimian type casting, there were two cases of type I, two cases of type III, one case of type IV, and one case of type II (postoperative recanalization). Of the 153 cases reported, the accuracy rate of the ultrasound diagnosis was $79.6 \%$, the misdiagnosis rate was $17.1 \%$, and the missed diagnostic rate was $3.3 \%$. In conclusion, echocardiography is the preferred method for non-invasive preoperative diagnosis of the ALVT and can accurately describe the type and involvement of the cardiac structure.
\end{abstract}

\section{Introduction}

Aortico-left ventricular tunnel (ALVT) is a rare congenital cardiac malformation, which accounts for only $0.12 \%$ of the incidence of congenital heart disease (1). ALVT refers to the abnormal channel between the ascending aorta and the

Correspondence to: Dr Qing Lv, Department of Ultrasound Imaging, Xiehe Hospital Affiliated to Tongji Medical College, Huazhong University of Science and Technology, 1277 Jiefang Road, Wuhan, Hubei 430022, P.R. China

E-mail:1vq2015@sina.com

Key words: echocariography, heart defects, congenital, aortico-left ventricular tunnel left ventricular and located at the side of ascending aorta valve. However, due to the unclear display of inlet and inside structure of the channel, missed diagnoses and misdiagnosis happened frequently in clinical treatment. The disease was first reported by Edwards in 1961 (1) and was termed ALVT by Levy et al in 1963 (2). Subsequently, few ALVT cases have been reported worldwide (3-7).

However, it has been found that transthoracic echocardiography (TTE) is a more effective way to display the inlet and inside structure of the channel clearly and correctly which plays an important role in diagnosing the ALVT $(6,7)$. In the present study, echocardiogram data of 6 ALVT patients in Xiehe Hospital Affiliated to Tongji Medical College (Hubei, China) have been analyzed to investigate the value of TTE on diagnosing ALVT.

\section{Materials and methods}

Subjects. Six patients with ALVT (4 males and 2 females, aged 3-41 years, with a median age of 26 years) were treated at the Xiehe Hospital Affiliated to Tongji Medical College hospital from June, 2007 to January, 2012. Clinical manifestations include heart murmur, chest tightness during activities, heart palpitations and diastolic murmur. The six patients underwent echocardiographic examination. One patient underwent a transesophageal echocardiography and another patient underwent a multi-slice CT. The surgery confirmed all manifestations.

The study was approved by the ethics committee of Tongji Medical College. Signed written informed consent was obtained from all the participants prior to the study.

Instruments and methods. The Philips iE33, GE Vivid 7 (GE Healthcare, Little Chalfont, Buckinghamshire,UK) and GE E9 color ultrasonic diagnostic apparatus (GE Healthcare) with the probe frequency of 3.5-7.5 MHz were used to conduct the study. The section was scanned with a two-dimensional ultrasound and color Doppler flow imaging (CDFI) and the parasternal left ventricle was observed in the long-axis view, the cardiac-base short-axis view of the aorta, the fivechamber view of the heart and the apical long axis view of the left ventricle, and the origin of the regurgitation beam was investigated. 
Table I. Echocardiographic features of 6 cases of ALVT.

\begin{tabular}{llclllllll}
\hline No. & Gender & Age (years) & Symptoms & AOP & AE & VSE & RVOTO & LVEF (\%) & CL \\
\hline 1 & Male & 21 & Chest distress & ARCS & - & - & - & 61 & AR, MR, VSD \\
2 & Male & 40 & No & AJRLCS & - & - & - & 65 & BAV, AS \\
3 & Female & 31 & Chest distress & ARCS & + & - & - & 40 & - \\
4 & Male & 3 & No & ARCS & - & + & - & 62 & - \\
5 & Male & 41 & Chest distress & ARCS & - & + & - & 61 & - \\
6 & Female & 12 & No & AJRLCS & + & + & - & 57 & AR, AVP \\
\hline
\end{tabular}

ALVT, aortico-left ventricular tunnel; AR, aortic regurgitation; MR, mitral regurgitation; VSD, ventricular septal defect; BAV, bicuspid aortic valve; AS, aortic stenosis; AVP, aortic valve prolapse; LVEF, left ventricular ejection fraction; AOP, aortic opening position; ARCS, above the right coronary sinus; AJRLCS, above the junction of right and left coronary sinus; AE, aortic expansion; VSE, ventricular septal expansion; RVOTO, right ventricular outflow tract obstruction; CL, complicated lesions.

\section{Results}

According to Hovaguimian et al, type casting, of the six confirmed cases, two were type I cases, two type III cases, one type IV cases, and one type II case (tunnel recanalization after ALVT operation) (3). The ultrasound results are shown in Table I.

According to the ALVT form, the ultrasound results of the six patients were divided into four types. Type I (two cases): i) from the two-dimensional echocardiography, we saw a tunnel structure in the aortic sinus (one case above the right coronary sinus, the other case above the junction of the left and right coronary sinus) of the parasternal left ventricle in the long-axis view, the cardiac-base short-axis view of the aorta, the five-chamber view of the heart and the apical long axis view of the left ventricle. It is connected to the aortic lumen through a narrow break (a case with the inner diameter of $0.65 \mathrm{~cm}$, the other with inner diameter of $0.8 \mathrm{~cm}$ ). The left ventricle tunnel flows through a break under the aortic valve ring down into the left ventricular outflow tract; ii) the CDFI and continuous Doppler ultrasound showed that a fine beam of regurgitation signal at a high speed could be seen during the period of tunnel diastole; iii) the aortic valve of one patient moved like a bicuspid with slight limitation of the normal opening and closing. The aortic valve of another case was normal in form and activities; iv) the ascending aorta was significantly widened or expanded and the left ventricle became bigger; and v) left and right coronary arteries had normal origins.

Type III (2 cases): i) from the two-dimensional echocardiography, we can see a small gap between the aortic root and the annulus in the cardiac-base short-axis view of the aorta and the five-chamber view of the heart. The gap was connected to a break between the aortic lumens on the aortic valve ring and entered through the lower aortic valve ring into the left ventricular outflow tract. According to the five-chamber view of the heart, the ventricular septum of the tunnel protruded like a tumor into the right ventricle. No continuous breaks were seen on the tumor wall (Fig. 1A); ii) the CDFI and continuous Doppler ultrasound showed a high-speed regurgitation signal entered through the tunnel into the left ventricular outflow tract (Fig. 1B) along the septal aneurysm tunnel wall; iii) the aortic valve of two cases was normal; iv) the blood in the right ventricular outflow tract flowed smoothly; v) the ascending aorta had widened or expanded and the left ventricle became bigger; and vi) left and right coronary arteries had healthy origins.

Type IV (1 case): i) the dimensional and transesophageal echocardiography showed that in the cardiac-base shortaxis view of the aorta and the five-chamber view of the heart, an abnormal tunnel between the aorta and the left ventricular outflow tract was evident. The aortic end was at the ventricle-artery connection of the left and right coronary sinus junction. It looked similar to a tumor protruding outside the heart and was connected to the break with the inner diameter of $0.8 \mathrm{~cm}$ in the aortic lumen. The left ventricular end of the tunnel was in the left ventricular outflow tract under the aortic valve. The ventricular septum of the tunnel protruded to the right ventricle resembling a tumor (Fig. 2A); ii) the CDFI and continuous Doppler showed that many high-speed regurgitation signals entered into the left ventricular outflow tract alongside the aortic aneurysm by the lateral wall aortic tumor of the tunnel through the aortic valve and along the septal aneurysm wall of the tunnel (Fig. 2B); iii) the right coronary aortic valve protruded to the left ventricular outflow tract during the diastole period. It opened normally but closed incorrectly; iv) the blood circulation was smooth in the right ventricular outflow tract; v) the ascending aorta was greatly widened and the left ventricle increased in size; and vi) left and right coronary arteries had normal origins.

Type II (1 case): this case was misdiagnosed as aortic regurgitation before surgery and later underwent an echocardiography exam because of heart failure and was diagnosed with ALVT. With surgery to repair the ALVT and replace the aortic valve, the tunnel achieved recanalization one year later. The ultrasonographs of the tunnel recanalization revealed the following: i) from the two-dimensional echocardiography, we could see that the aorta was connected to the left ventricle outflow tract by the tunnel in the parasternal left ventricle in the long-axis view, the cardiac-base short-axis view of the aorta, the five-chamber view of the heart and the apical long axis view of the left ventricle. The aortic side of the tunnel had a large opening and the aortic wall protruded outside the heart like tumors. The left ventricular end of the tunnel was connected to the left ventricular outflow tract under 

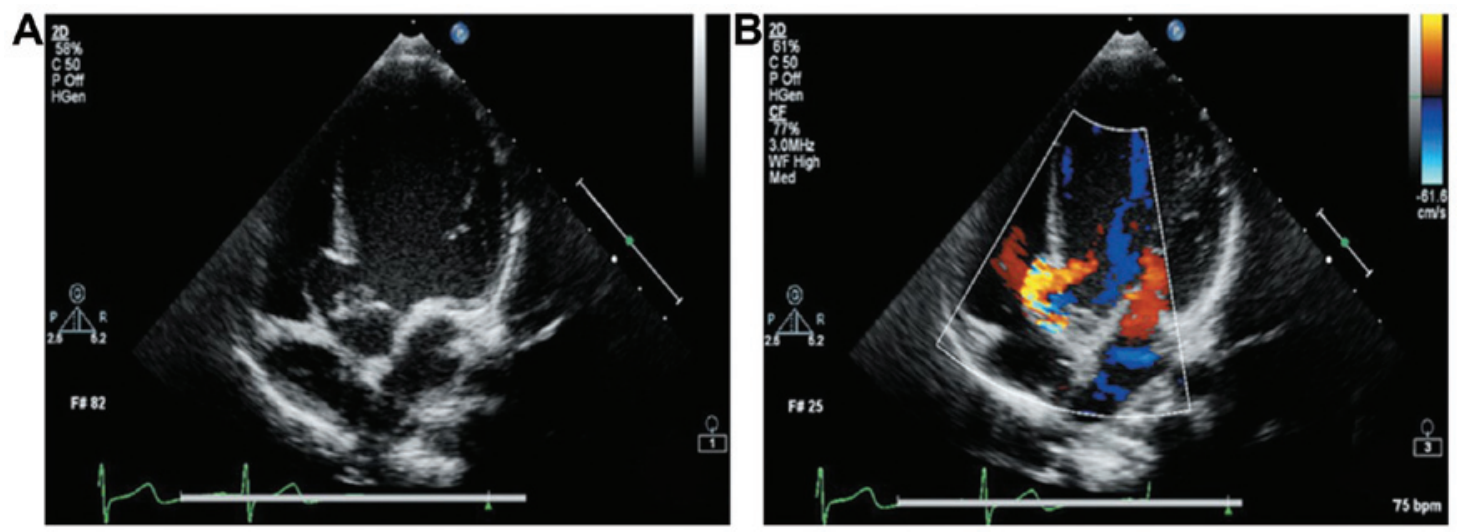

Figure 1. Hovaguimian type III ultrasonogram. (A) Ventricular septal expansion of ALVT; (B) regurgitation signals seen in the tunnel during the period of diastole. ALVT, aortico-left ventricular tunnel.
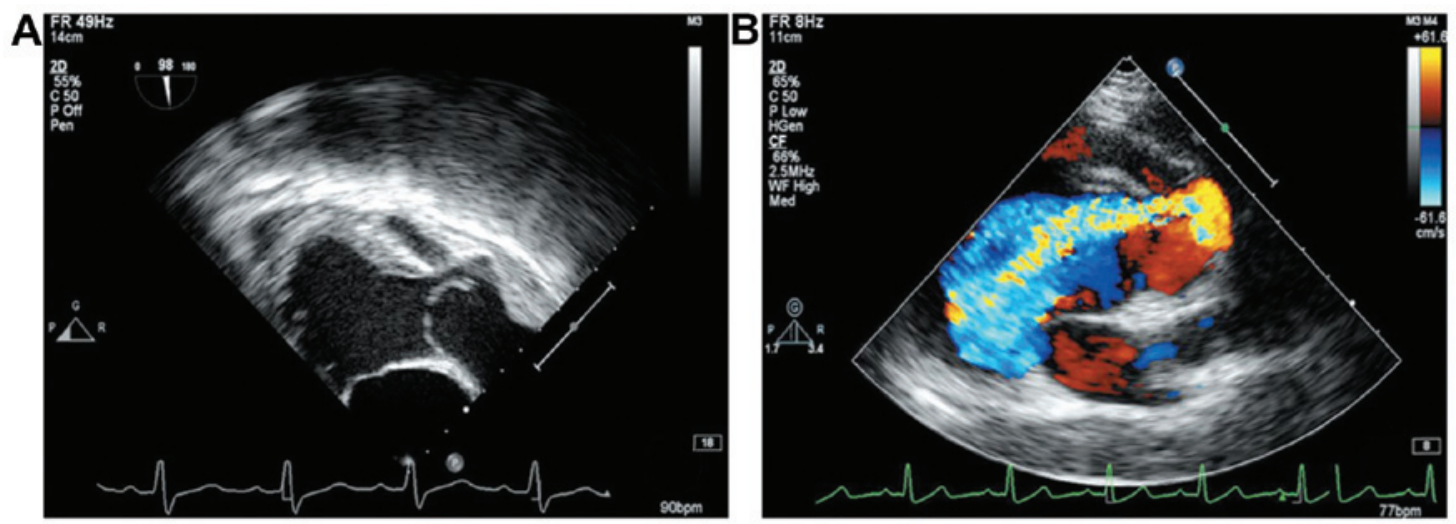

Figure 2. Hovaguimian IV sonogram, the mixed type of II and III. (A) The transesophageal echocardiography shows the aortic sinus wall and ventricular septal aneurysm expansion inside the tunnel; (B) the tunnel Shu. (B) A lot of regurgitation signals flow via the tunnel into the left ventricle.

the aortic valve ring through a small gap; ii) the CDFI and continuous Doppler ultrasound showed that an appropriate amount of high-speed diastolic regurgitation signals entered into the left ventricular outflow tract through the tunnel along the aortic tumor wall; iii) the strong echo of the artificial metal aortic valve at the position of the aortic valve could be heard. Its form and activities were normal; iv) the ascending aorta was widened and the left ventricle became larger. The left ventricular blood ejection fraction measured by M-mode echocardiography was at $40 \%$; and v) the origins of the left and right coronary arteries were normal.

The six patients were diagnosed correctly and confirmed by surgery. The postoperative echocardiography showed that, except for one case of recanalization, the re-examination one month after the surgery witnessed no or only a small amount of residual regurgitation signals. The left ventricular blood ejection fraction remained normal.

\section{Discussion}

Cause of ALVT. The cause of ALVT is uncertain. Cooley et al (4) argued that it may be similar to the increased deposits of acid mucopolysaccharides in the arteries of patients affected with Marfan syndrome. However, Terada et al believed that the tunnel formation was the result of the abnormal development of the right coronary artery based on three fetal autopsies (5).
Histological sections showed that the aortic end of the tunnel was the arterial structure and linked closely or even fused with the origin of the right coronary artery. Levy et al also suspected that ALVT belonged to abnormalities of the coronary artery (2). It was reported that as the coronary artery originated from ALVT, that ALVT was a special type of coronary artery fistula (6-8).

Clinical features of ALVT. According to the clinical data of 153 cases of ALVT reported in relevant literature (9-14), ALVT has the following characteristics: i) there are more male than female patients (82/23), 1 day to 71 years of age; ii) the tunnel is mostly single; iii) greatly closing aortic valves lead to regurgitation while frequent closing leads to prolapse; iv) cardiac malformations are often manifested in bicuspid valves and sometimes in anomalous origins of the coronary artery (15-18) or a single coronary artery; v) patients with no other cardiac malformations and complications visit the doctor when they discover a diastolic murmur during a medical examination. Patients with small tunnels have no clinical manifestations at the early stage but show palpitation, shortness of breath, and other symptoms of cardiac insufficiency after activities due to an overload of the left ventricular volume.

Comparison of the advantages and disadvantages of angiography and echocardiography in the diagnosis of ALVT. 
Before echocardiography is used, selective angiography of the ascending aorta or aortic openings of the tunnel is the golden standard for diagnosing this disease (19-21). Successful angiography of the ascending aorta can diagnose the disease, but the technology can only be dependent on indirect signs from the contrast agent reflux. However, when the coexisting aortic valves cannot close completely, the ALVT contrast agents reflux is concealed. Thus, its clinical application has certain restrictions. Echocardiography can clearly show ALVT in two-dimensional images in real time, and CDFI can display the blood flow signal within ALVT. Echocardiography is non-invasive, accurate, and simple to use and has become the standard imaging exam for diagnosing ALVT (22-24). Of the 153 cases reported, the accuracy rate of the ultrasound diagnosis was $79.7 \%$, the misdiagnosis rate was $17.0 \%$, and missed diagnosis rate was $3.3 \%$.

Key points, symptoms, complicated lesions and differential diagnosis of the ultrasound diagnosis of ALVT. i) Key points and symptoms of the ultrasound diagnosis of ALVT: a) common scanning sections include the parasternal left ventricle in the long-axis view, the cardiac-base short-axis view of the aorta, the apical five-chamber view of the heart and the apical long axis view of the heart. To uncover aortic valve regurgitation, great attention should be paid to the multi-slice scanning to identify the origin of the regurgitation beam; b) The position of aortic opening of ALVT is relatively complex, and more common at the ventricle-artery connection $(68.0 \%)$ to which the right ventricular coronary valve attaches or perhaps at the ventricle-artery connection to which the left coronary aortic valve $(10.4 \%)$ or non-coronary valve (5.9\%) attaches, or the triangle (13.1\%) of active sinus bicuspid valves or in the ascending aorta (2.6\%); c) ALVT type casting: Hovaguimian et al divided ALVT into four types (3). Type I is simply ALVT whereby the aortic opening is small and cracked, and is not associated with aortic valve damage. In type II, the aortic opening is oval and the corresponding aortic sinus aneurysm wall expands like tumors with or without aortic valve damage. As for type III, the septal heart aneurysm of the tunnel expands with or without the right ventricular outflow tract obstruction (25). Type IV is a mix of types II and III. In this group, case 1 and 2 belong to type I, case 3 to type II, case 4 and 5 to type III, case 6 to type IV. Ninety-three of the 153 cases reported here, can be categorized into 34 cases of type I (36.62\%), 40 cases of type II (43.0\%), 14 cases of type III (15.0\%), 5 cases of type IV (5.4\%); d) color and spectral Doppler displays that high-speed regurgitation signal can be seen in the tunnel between the aorta and the left ventricle during the diastole period. The regurgitant beam starts from the aortic valve; e) long-term high-speed blood flow impacting the aortic valve and the left ventricular outflow tract easily leads to aortic valve insufficiency and aortic valve prolapse; f) whether the origin of the left and right coronary arteries is normal should be made clear; g) of 153 cases reported here, there are only four cases of recanalization after ALVT surgery. The ALVT ultrasonography of partial recanalization is similar to perivalvular leakage.

ii) According to the clinical data of the 153 cases, there are 51 cases (33.3\%) with aortic regurgitation and 19 cases (12.4\%) with coronary artery abnormalities. Other lesions include bicuspid aortic valve malformation, pulmonary stenosis, aortic stenosis, ventricular septal defect, the main aortic valve prolapse (26).

iii) Differential diagnosis of LVT: In the 17 misdiagnosed cases reported in the literature, seven cases were misdiagnosed as aortic valve insufficiency, two cases as Valsalva sinus aneurysm, one case as coronary artery, left ventricular fistula, one case as ventricular septal defect with aortic malformations, one case as four-leaf aortic valve, two cases as aortic valve prolapse, one case as infective endocarditis, one case as paravalvular abscess, one case as Marfan syndrome. It is harder to differ the Valsalva sinus aneurysm and coronary artery, left ventricular fistula from ALVT. We believe that sinus of Valsalva aneurysm usually takes the form of a pocket pouch or petals. The enormous influx of arterial blood into the left ventricle during the diastole period can increase the sinus volume, and flow into the left ventricular outflow tract. The two-dimensional image shows that the sinus wall is interrupted. Remnants of the tumor wall tissue at the edge of the break are waving like valves. Blood flows through the break into the left ventricle whose shunt flow signals originate in the Valsalva sinus below the level of the annulus. ALVT often originates from above the opening of the coronary artery (6). The aortic valve is like a tunnel or an ampulla. The regurgitant beam originates above the level of annulus. The coronary artery of patients with coronary artery, left ventricular fistula expands while that of patients with ALVT no abnormity is observed.

In conclusion, echocardiography is the preferred method for the non-invasive preoperative diagnosis of ALVT and can accurately describe the type and the involvement of the cardiac structure.

\section{References}

1. Edwards JE: An atlas of acquired disease of the heart and great vessels. Vol 2. 2nd edition. WB Saunders, Philadelphia, p1142, 1961.

2. Levy MJ,Lillehei CW, Anderson RC, Amplatz K and Edwards JE: Aortico-left ventricular tunnel. Circulation 27: 841-853, 1963.

3. Hovaguimian H, Cobanoglu A and Starr A: Aortico-left ventricular tunnel: a clinical review and new surgical classification. Ann Thorac Surg 45: 106-112, 1988.

4. Cooley RN, Harris LC and Rodin AE: Abnormal communication between the aorta and left ventricle; aortico-left ventricular tunnel. Circulation 31: 564-571, 1965.

5. Terada T, Sakurai H, Nonaka T, Sakurai T, Sugiura J and Otsuka R: Surgical repair of aortic regurgitation with left ventricular aneurysm diagnosed preoperatively as aortico-left ventricular tunnel. World J Pediatr Congenit Heart Surg 5: 583-585, 2014.

6. Martins JD, Sherwood MC, Mayer JE Jr and Keane JF: Aortico-left ventricular tunnel: 35-year experience. J Am Coll Cardiol 44: 446-450, 2004.

7. Cook AC, Fagg NL, Ho SY, Groves AM, Sharland GK, Anderson RH and Allan LD: Echocardiographic-anatomical correlations in aorto-left ventricular tunnel. Br Heart J 74: 443-448, 1995.

8. Hucin B, Horvath P, Skovránek J, Reich O and Samánek M: Correction of aortico-left ventricular tunnel during the first day of life. Ann Thorac Surg 47: 254-256, 1989.

9. Huang XS, Hou YS, Feng B, He Y, XU Y and Huang ZG: Transthoracic and transesophageal echocardiography in the diagnosis of aortico-left ventricular tunnel. Chinese J Ultrasound Med 20: 304-307, 2004 (In Chinese).

10. Zhang HB, Xu ZW, Su ZZ and Ding WX: Diagnosis and surgical treatment of aortico-left ventricular tunnel. Chinese J Thoracic Cardiovas Surg 20: 268-270, 2004 (In Chinese). 
11. Hou CJ, Deng DA, Zhu XY, Han XM, Wang QG, Sheng XT, Jin Y, Cui CS and Zhang P: A study on imaging characteristics of color Doppler echocardiography of aortic left ventricular tunnel. J China Clin Med Imaging 17: 34-36, 2006 (In Chinese).

12. Horváth P, Balaji S, Skovránek S, Hucin B, de Leval MR and Stark J: Surgical treatment of aortico-left ventricular tunnel. Eur J Cardiothorac Surg 5: 113-116, discussion 117, 1991.

13. Wang Q, Qiu LC, Shi HY and Wang LX: One case of advanced aortico-left ventricular tunnel. Chinese J Thoracic Cardiovas Surg 22: 307, 2006 (In Chinese).

14. Grünenfelder J, Zünd G, Prêtre R, Schmidli J, Vogt PR and Turina MI: Right coronary artery from aorto-left ventricular tunnel: case report of a new surgical approach. J Thorac Cardiovasc Surg 116: 363-365, 1998.

15. Zhu J, Jiang Z, Huang J, Wu S and Mei J: Aortico-left ventricular tunnel arising from the noncoronary sinus associated with a ventricular septal defect. Ann Thorac Surg 98: e135-e137, 2014.

16. Pockett CR, Chan S and Smallhorn J: Aortico-left ventricular tunnel: the elusive diagnosis. Pediatr Cardiol 34: 1743-1745, 2013.

17. Smith BM, Cochran CD and Owens ST: Aortico-left ventricular tunnel and left ventricular non-compaction: a case series. Cardiol Young 26: 1-4, 2015.

18. Thomas E, Maskari S and Farqani A: Percutaneous device closure of aortico-left ventricular tunnel using Amplatzer vascular plug III. Cardiol Young 23: 755-758, 2013.
19. Paech C, Pfeil N, Wagner R, Kostelka M and Weidenbach M: Smart nature. Aortico-left ventricular tunnel bypassing congenital critical aortic stenosis. Echocardiography 30: E344-E345, 2013.

20. Shiraishi S, Takahashi M, Watanabe M and Tsuchida M: Surgical repair of aortico-left ventricular tunnel: report of two cases. Asian Cardiovasc Thorac Ann 21: 67-70, 2013.

21. Cebeci M, Kalayci S, Demirkan BM, Guray YA and Tufekcioglu O: Aortico-left ventricular tunnel with uncommon origin. Eur Heart J Cardiovasc Imaging 15: 943, 2014.

22. Li X, Zhao R, Liu B, Wang W, Yu Y, Shi X and Xu Y: Noninvasive imaging evaluation of aortico-left ventricular tunnel: a case report. Zhonghua Xin Xue Guan Bing Za Zhi 42: 345-346, 2014 (In Chinese)

23. Song L, Jin J and Tao L: Surgical correction of an aortico-left ventricular tunnel originating from the left aortic sinus with a left coronary artery anomaly. J Card Surg 30: 108-110, 2015.

24. Bash SE, Huhta JC, Nihill MR, Vargo TA and Hallman GL: Aortico-left ventricular tunnel with ventricular septal defect: two-dimensional/Doppler echocardiographic diagnosis. J Am Coll Cardiol 5: 757-760, 1985.

25. Nezafati MH, Maleki MH, Javan $\mathrm{H}$ and Zirak N: Repair of aorto-left ventricular tunnel arising from the left sinus of valsalva. J Card Surg 25: 345-346, 2010.

26. Myers JL and Mehta SM: Congenital Heart Surgery Nomenclature and Database Project: Aortico-left ventricular tunnel. Ann Thorac Surg 69 (Suppl 4): S164-S169, 2000 